\title{
Shantala para promoção da saúde e conforto de bebês: revisão de literatura
}

\author{
Shantala for the promotion of health and comfort of babies: literature \\ review
}

Shantala para la promoción de la salud y la comodidad de los bebés: revisión de literatura

Telma Vitorina Ribeiro-Lima ${ }^{1 *}$, Lília lêda Chaves Cavalcante².

\section{RESUMO}

Objetivo: Explorar recente produção acerca dos benefícios da Shantala para bebês em contextos domésticos e de cuidado coletivo. Métodos: Trata-se de uma revisão integrativa cujos critérios de inclusão consideraram o uso terapêutico da Shantala e a avaliação de bebês que a tenham recebido. Pesquisando o descritor "Shantala" nas bases de dados Pubmed e Scielo obteve-se um único artigo; enquanto na BVS, mais seis. Resultados: Os artigos evidenciaram que, após a Shantala, as crianças estavam mais tranquilas e relaxadas, com melhor qualidade de sono, além de apresentarem alívio de cólicas e melhor funcionamento intestinal, diferença significativa na frequência cardíaca, frequência respiratória e cortisol salivar e estreitamento do vínculo afetivo com mãe e familiares. Crianças portadoras de Síndrome de Down, além dos benefícios antes citados, apresentaram melhora do tônus muscular dos braços, do controle cervical e deambulação sem apoio. Os desenhos dos estudos evidenciaram necessidade de elaboração e descrição mais detalhada da metodologia, com vistas a reproduções futuras para comparações entre sujeitos e populações em contextos diversos. Considerações Finais: Tais intervenções se constituem como recursos terapêuticos que podem reduzir o estresse em bebês, auxiliando-os nos aspectos psicológicos, motor, físico e emocional de seu desenvolvimento, em qualquer contexto em que se encontre.

Palavras-chave: Recém-nascido, Massagem, Saúde da criança, Serviços de saúde da criança, Literatura de revisão como assunto.

\begin{abstract}
Objective: This review aims to explore recent production on the benefits of Shantala for babies in domestic and collective care settings. Methods: It is an integrative review whose inclusion criteria considered the therapeutic use of Shantala and the evaluation of infants who received it. Searching the descriptor "Shantala" in the Pubmed and Scielo databases, we obtained a single article; while at the VHL, six more. Results: The articles showed that after Shantala, the children were calmer and more relaxed, with better sleep quality, with colic relief and better bowel functioning, significant difference in heart rate, respiratory rate and salivary cortisol, and affective bonding with mother and mother. relatives. Children with Down Syndrome, in addition to the benefits mentioned above, showed improvement in arm muscle tone, cervical control and unaided walking. The study designs evidenced the need for elaboration and more detailed description of the methodology, with a view to future reproductions for comparisons between subjects and populations in different contexts. Final Considerations: These interventions constitute therapeutic resources that can reduce stress in babies, assisting them in the psychological, motor, physical and emotional aspects of their development, in any context in which they are found.
\end{abstract}

Key-words: Newborn, Massage, Child health, Child health services, Review literature as topic.

\section{RESUMEN}

Objetivo: Esta revisión tiene como objetivo explorar la producción reciente sobre los beneficios de Shantala para los bebés en entornos de los cuidados doméstico y colectivo. Métodos: Es una revisión integradora cuyos los criterios de la inclusión consideraron el uso terapéutico de Shantala y la evaluación de los recién

${ }^{1}$ Universidade Federal do Pará (UFPA). Belém - Pará. *E-mail: telmavitorina@gmail.com

Este estudo foi financiado pela Coordenação de Aperfeiçoamento de Pessoal de Nível Superior (CAPES) processo no 23038.013648/2018-51

SUBMETIDO EM: 11/2019

ACEITO EM: 12/2019

PUBLICADO EM: 5/2020

REAS/EJCH | Vol.Sup.n.48 | e2375 | DOI: https://doi.org/10.25248/reas.e2375.2020 Página 1 de 9 
nacidos que lo recibieron. Al buscar el descriptor "Shantala" en las bases de datos Pubmed y Scielo, obtuvimos un solo artículo; mientras que en la BVS, seis más. Resultados: Los artículos mostraron que después de la Shantala, los niños estaban más tranquilos y relajados, con mejor calidad del sueño, alivio del cólico y mejor funcionamiento intestinal, diferencia significativo en la frecuencia cardíaca, la frecuencia respiratoria y el cortisol salival, y el vínculo afectivo con la madre y miembros de la familia. Los niños con síndrome de Down, además de los beneficios mencionados anteriormente, mostraron una mejora en el tono muscular del brazo, el control cervical y la marcha sin ayuda. Los diseños del estudio evidenciaron la necesidad de elaboración y una descripción más detallada de la metodología, con miras a futuras reproducciones para realizar comparaciones entre sujetos y poblaciones en diferentes contextos. Consideraciones Finales: Estas intervenciones constituyen recursos terapéuticos que pueden reducir el estrés en los bebés, ayudándolos en los aspectos psicológicos, motores, físicos y emocionales de su desarrollo, en cualquier contexto en el que se encuentren.

Palabras-claves: Recién Nacido, Massaje, Salud del niño, Servicios de salud del niño, Literatura de revisión como asunto.

\section{INTRODUÇÃO}

As práticas de saúde das comunidades tradicionais, de modo especial às massagens, vêm ganhando cada vez mais destaque e adeptos não apenas no seio da sociedade urbana, mas também entre a comunidade científica (NARDO LRO, et al., 2014). De modo geral, elas se constituem em recursos terapêuticos de comprovada efetividade e custos relativamente mais baixos. Dentre essas técnicas há aquelas que podem beneficiar grupos mais específicos, como a Shantala que é direcionada ao público infantil, principalmente em seus primeiros meses de vida (RIBEIRO-LIMA TV, et al., 2018, SOUZA NR, et al., 2011).

A massagem Shantala se constitui em um conjunto de movimentos aplicados ao corpo do bebê com o auxílio de óleo vegetal de boa qualidade e procedência, incluindo amassamentos e mobilizações articulares. Sua história tem origem nas práticas tradicionais indianas repassadas essencialmente de mãe para filha como algo comum e corriqueiro em sua rotina diária. No entanto, na década de 1970 foi apresentada ao Ocidente pelo relato sensível e minucioso do médico obstetra francês Frédérick Leboyer, que, durante um período de imersão na cultura indiana teve a oportunidade de observar uma jovem que aplicava essa massagem em seu bebê e cujo nome, Shantala, batizou a técnica então descrita por Leboyer (LEBOYER F, 1995). Com base em dados de estudos já publicados, é possível afirmar que técnicas de massagem e estimulação tátil/cinestésica podem favorecer o desenvolvimento motor e do tônus muscular da criança, bem como sua propriocepção. (BRÊTAS J, SILVA M, 1998, CARVALHO R, et al., 2010; CRUZ C, CAROMANO F, 2005, 2007; FIELD T, et al., 1986).

Uma revisão recente elencou alguns estudos que demonstraram os benefícios de se aplicar intervenções de massagens infantis e estimulações táteis em bebês que viviam em ambientes de cuidado coletivos, tais como hospitais e creches (RIBEIRO-LIMA TV, CAVALCANTE LIC, 2019). Benefícios semelhantes podem ser alcançados por meio da massagem indiana Shantala (CARVALHO R, et al., 2010). A técnica também pode melhorar o trânsito intestinal dos bebês e aliviar os sintomas de cólicas abdominais, muito comuns nesse período (BRÊTAS J, SILVA M, 1998, UMEMURA JF, et al., 2010).

Levando-se em consideração a relevância de se encorajar cada vez mais propostas de intervenção que possam elevar o bem estar e promover o desenvolvimento de crianças em quaisquer contextos em que se encontrem inseridas, bem como o potencial real da massagem Shantala em cumprir esse papel, traz-se aqui o objetivo explorar o que vem sendo produzido acerca dos benefícios da Shantala para populações pediátricas em contextos domésticos e de cuidado coletivo.

\section{MÉTODOS}

Para uma melhor elucidação dos benefícios da Shantala para bebês em ambientes domésticos e de cuidado coletivo, o presente trabalho traz uma revisão integrativa de literatura de caráter exploratório dentro dessa temática. Como critérios de inclusão foram considerados: (1) ter utilizado a Shantala como recurso terapêutico para bebês; (2) ter avaliado os bebês que receberam a Shantala. Como critérios de exclusão, 
foram considerados: (1) não ter utilizado a Shantala como recurso terapêutico para bebês; (2) não ter avaliado os bebês que receberam a Shantala.

Efetuou-se uma pesquisa com o descritor "Shantala" nas bases de dados PubMed [ferramenta de busca do Sistema Online de Busca e Análise de Literatura Médica (do inglês: Medical Literature Analysis and Retrieval System Online - MEDLINE®)] e Scientific Electronic Library Online (Scielo), a qual trouxe um único artigo que tratava sobre o assunto e que foi realizado em uma creche pública em São Paulo, Brasil (FOGAÇA MDC, et al., 2005).

A seguir, o mesmo descritor foi pesquisado na Biblioteca Virtual em Saúde (BVS), a partir da qual apenas seis artigos contemplavam o tema, igualmente todos realizados em território brasileiro. A busca nas três bases foi realizada no dia 11 de outubro de 2018 e os artigos na íntegra foram baixados entre esta data e o dia 5 de novembro de 2018 e armazenados no software Mendeley ${ }^{\circledR}$ para posterior leitura de seu conteúdo.

Essa busca resultou em 16 artigos, dos quais 7 foram aproveitados por contemplarem os critérios de inclusão. Os 9 restantes foram excluídos, sendo que 4 não contemplaram os critérios de inclusão e 5 eram repetidos. Os sete artigos incluídos foram lidos na íntegra e avaliados de acordo com os contextos onde foram realizados, as metodologias utilizadas e seus respectivos resultados. Os artigos serão descritos a seguir para que se possa visualizar um panorama do que tem sido publicado em literatura científica acerca do uso da Shantala em bebês em contextos de cuidado coletivo.

No decorrer do texto, os estudos serão abordados com base nos contextos e ambientes em que foram realizados e serão discutidos de acordo com as metodologias utilizadas e os resultados obtidos. As características mais relevantes para comparação serão apresentadas em tabelas, cada uma abordando um grupo de informações: (1) características da população estudada; (2) informações sobre as metodologias utilizadas; (3) contextos e benefícios de cada estudo. Para melhor visualização, os artigos estarão dispostos em ordem alfabética de seus primeiros autores.

\section{RESULTADOS E DISCUSSÃO}

O Quadro 1 traz a identificação dos artigos com informações que os caracterizam, tais como: características clínicas, tamanho em números $(\mathrm{N})$ e faixa etária da população estudada, e ainda cidade, Estado, contexto e ambiente onde os estudos ocorreram.

Quadro 1 - Artigos de acordo com a condição clínica e faixa etária da população estudada, tamanho da amostra e cidade/Estado onde os estudos foram realizados.

\begin{tabular}{|l|l|c|c|c|}
\hline Autor(es)/ano & População & N & Faixa etária & Cidade/Estado \\
\hline $\begin{array}{l}\text { BARBOSA KC, et } \\
\text { al., 2011 }\end{array}$ & Crianças com Síndrome de Down & 3 & $1-3$ anos & Itaquaquecetuba, SP \\
\hline $\begin{array}{l}\text { CARVALHO R, et } \\
\text { al., 2010 }\end{array}$ & Crianças com Síndrome de Down & 1 & 2 anos & Poços de Caldas, MG \\
\hline $\begin{array}{l}\text { FOGAÇA MDC, et } \\
\text { al., 2005 }\end{array}$ & $\begin{array}{l}\text { Bebês saudáveis em um berçário } \\
\text { público }\end{array}$ & 11 & $4-6$ meses & São Paulo, SP \\
\hline $\begin{array}{l}\text { LINKEVIEIUS } \\
\text { TAK, et al., 2012 }\end{array}$ & $\begin{array}{l}\text { Lactentes saudáveis em uma creche } \\
\text { Crianças com e sem riscos de }\end{array}$ & 9 & $10,22 \pm 1,3$ meses & Araras, SP \\
\hline $\begin{array}{l}\text { MOREIRA NRTL, } \\
\text { et al., 2011 }\end{array}$ & $\begin{array}{l}\text { distúrbios no desenvolvimento motor } \\
\text { (prematuridade e disfunção motora) }\end{array}$ & 3 & $4-12$ meses & João Pessoa, PB \\
\hline $\begin{array}{l}\text { MOTTER AA, } \\
\text { et al., 2012 }\end{array}$ & Crianças & 9 & 16 meses (média) & Matinhos, PR \\
\hline $\begin{array}{l}\text { SOUZA NR, et al., } \\
\text { 2011 }\end{array}$ & Bebês de mães primíparas & 7 & $1-6$ meses & Passos, MG \\
\hline
\end{tabular}

Fonte: Ribeiro-Lima TV e Cavalcante LIC, 2019. 
Por sua vez, o Quadro 2 mostra informações sobre a metodologia aplicada em cada um dos estudos que elegeram a Shantala como alternativa de intervenção na rotina de bebês. Este quadro descreve quem aplicou a Shantala, qual o número de aplicações $(\mathrm{N})$, a duração das sessões em minutos, quantas aplicações foram realizadas por dia e por semana e qual o período de duração da intervenção.

Quadro 2 - Estudos sobre Shantala relacionados de acordo com quem a aplicou, quantidade e duração das aplicações e sua distribuição ao longo do tempo.

\begin{tabular}{|c|c|c|c|c|c|c|}
\hline & & & Aplicaçõe & & & \\
\hline Autor(es)/ano & Quem aplicou & $\mathbf{N}$ & $\begin{array}{r}\text { Duração } \\
\text { (minutos) }\end{array}$ & Por dia & $\begin{array}{c}\text { Por } \\
\text { semana }\end{array}$ & Período \\
\hline $\begin{array}{l}\text { BARBOSA KC, et al., } \\
2011\end{array}$ & $\begin{array}{l}\text { Pais/ } \\
\text { cuidadores }\end{array}$ & 8 & 20 & -- & 1 & 60 dias \\
\hline $\begin{array}{l}\text { CARVALHO R, et al., } \\
2010\end{array}$ & Não ficou claro & 20 & 50 & -- & -- & -- \\
\hline $\begin{array}{ll}\text { FOGAÇA } & \text { MDC, } \\
\text { et al., 2005 } & \\
\end{array}$ & A autora & 3 & 15 & 1 & 3 & $\begin{array}{l}1 \\
\text { semana }\end{array}$ \\
\hline $\begin{array}{l}\text { LINKEVIEIUS } \\
\text { TAK, et al., } 2012\end{array}$ & A pesquisadora & 1 & 30 & 1 & 1 & 1 dia \\
\hline $\begin{array}{l}\text { MOREIRA NRTL, } \\
\text { et al., } 2011\end{array}$ & Mães & $\begin{array}{l}6 \\
\text { (média) }\end{array}$ & -- & -- & -- & -- \\
\hline $\begin{array}{l}\text { MOTTER AA, et al., } \\
2012\end{array}$ & Discentes & $\begin{array}{l}15 \\
\text { (média) }\end{array}$ & -- & -- & 2 & -- \\
\hline $\begin{array}{l}\text { SOUZA NR, et al., } \\
2011\end{array}$ & Não ficou claro & - & -- & -- & -- & -- \\
\hline
\end{tabular}

Fonte: Ribeiro-Lima TV e Cavalcante LIC, 2019.

Por fim, o Quadro 3 exibe os principais benefícios registrados nestes estudos que puderam favorecer as crianças que receberam sessões de Shantala em diversos contextos e ambientes. Como é possível se observar nos Quadros 1 e 2, dois estudos envolveram crianças portadoras de Síndrome de Down (SD) triadas nas Associações de Pais e Amigos dos Excepcionais (APAE) de suas cidades. No primeiro, as três crianças com idade entre 1 e 3 anos receberam a massagem de seus pais e/ou cuidadores, no entanto não fica claro se o procedimento é realizado na APAE (ambulatorial) ou na residência da família (domiciliar) (BARBOSA KC, et al., 2011). Por outro lado, no segundo, a única criança avaliada, de dois anos de idade, recebeu a Shantala no ambiente domiciliar, ainda que não fique evidente quem tenha aplicado: fossem os pais ou a equipe de pesquisa (CARVALHO R, et al., 2010).

Conforme se pode ver no Quadro 3, em se tratando das metodologias utilizadas, os dois estudos envolvendo as crianças com SD não apresentaram outra semelhança além da aplicação da Shantala. No estudo realizado em ambiente de cuidado coletivo, a massagem foi aplicada pelos pais ou cuidadores, sendo uma sessão semanal de vinte minutos cada no decorrer de oito semanas, totalizando oito sessões dentro de um período de sessenta dias (BARBOSA KC, et al., 2011).

Por outro lado, no estudo realizado em contexto domiciliar foram aplicadas vinte sessões de Shantala, com duração de cinquenta minutos cada uma, no entanto não ficou claro quem aplicou e quantas aplicações diárias ou semanais e nem o período de duração das aplicações ao longo do tempo (CARVALHO R, et al., 2010).

No estudo com as três crianças com SD em contexto de cuidado coletivo, os resultados foram avaliados a partir de dois questionários, um ao início, para se obtiver maiores informações sobre as crianças e outro ao final, para se verificar os resultados da técnica utilizada (BARBOSA KC, et al., 2011).

Por sua vez, a única criança com SD acompanhada em contexto doméstico recebeu avaliação neurológica pela escala de Amiel-Tison (FONSECA LF, et al., 2002) e a partir de um questionário a respeito do padrão de desenvolvimento de crianças portadoras da síndrome de Down (LIMA PLS, 2004) antes e após o tratamento (CARVALHO R, et al., 2010). Os principais resultados de ambos os estudos estão descritos a seguir e estão sintetizados no Quadro 3. 
Quadro 3 - Benefícios da aplicação da massagem Shantala em bebês descritos em artigos científicos.

\begin{tabular}{|l|l|l|}
\hline Artigo & Contexto & $\begin{array}{l}\text { Benefícios descritos a partir da aplicação de } \\
\text { sessões de Shantala em crianças }\end{array}$ \\
\hline $\begin{array}{l}\text { BARBOSA KC, et al., } \\
2011\end{array}$ & $\begin{array}{l}\text { Cuidado } \\
\text { coletivo }\end{array}$ & $\begin{array}{l}\text { Redução do estado de agitação (crianças mais calmas e } \\
\text { tranquilas) } \\
\text { Maior disposição para brincar } \\
\text { Melhora na qualidade do sono } \\
\text { Uma criança começou a andar sem apoio } \\
\text { Mais afetivo e carinho entre mãe e filho } \\
\text { Melhor desenvolvimento psicomotor } \\
\text { Maior envolvimento dos responsáveis }\end{array}$ \\
\hline $\begin{array}{l}\text { CARVALHO R, et al., } \\
2010\end{array}$ & Domiciliar & $\begin{array}{l}\text { Melhora no tônus muscular dos membros superiores } \\
\text { (MMSS) } \\
\text { Melhora do controle cervical } \\
\text { Melhora na qualidade do sono } \\
\text { Aumento no padrão motor de 36\% para 60\% dos itens } \\
\text { avaliados } \\
\text { Melhora no manuseio e convívio da mãe com a criança } \\
\text { Melhora no controle das pernas }\end{array}$ \\
\hline $\begin{array}{l}\text { FOGAÇA MDC, et al., } \\
2005\end{array}$ & $\begin{array}{l}\text { Cuidado } \\
\text { coletivo }\end{array}$ & $\begin{array}{l}\text { Modificação nos valores de cortisol salivar } \\
\text { Possível adaptação do eixo HPA e do ritmo } \\
\text { circadiano }\end{array}$ \\
\hline $\begin{array}{l}\text { LINKEVIEIUS TAK, et al., } \\
2012\end{array}$ & $\begin{array}{l}\text { Cuidado } \\
\text { coletivo }\end{array}$ & $\begin{array}{l}\text { Diferença significativa na frequência respiratória e na } \\
\text { frequência cardíaca }\end{array}$ \\
\hline $\begin{array}{l}\text { MOREIRA NRTL, et } \\
\text { al., 2011 }\end{array}$ & $\begin{array}{l}\text { Cuidado } \\
\text { coletivo }\end{array}$ & $\begin{array}{l}\text { Crianças mais tranquilas } \\
\text { Melhora na qualidade do sono } \\
\text { Maior aproximação e melhora da afetividade no vínculo } \\
\text { mãe-filho }\end{array}$ \\
\hline $\begin{array}{l}\text { Relaxamento dos bebês } \\
\text { Incentivo à criação e fortalecimento de vínculo com } \\
\text { quem aplica a Shantala } \\
\text { Crianças mais tranquilas quando recebiam Shantala } \\
\text { de seus pais em casa }\end{array}$ \\
\hline $\begin{array}{l}\text { Culivio de cólicas } \\
\text { Equilíbrio no funcionamento intestinal } \\
\text { Melhora no padrão de sono } \\
\text { Tranquilidade e relaxamento } \\
\text { Fortalecimento do vínculo familiar }\end{array}$ \\
\hline
\end{tabular}

Fonte: Ribeiro-Lima TV e Cavalcante LIC, 2019.

Dentre os resultados observados no primeiro estudo, foram registrados: a redução do estado de agitação das crianças, de modo que elas se tornaram menos inquietas e mais calmas e tranquilas, maior disposição para brincar, melhora da qualidade do sono e uma das crianças começou a andar sem apoio, segundo relato dos autores. Ao final do estudo, a equipe de pesquisa pôde verificar que a técnica Shantala promoveu um contato mais afetivo e de maior carinho entre as mães e as crianças com SD, bem como um melhor desenvolvimento psicomotor das crianças. A melhora do laço entre as mães e suas crianças incluiu ainda o maior envolvimento do responsável com a criança, fator que fortalece o vínculo parental (BARBOSA KC, et al., 2011).

Enquanto os resultados do segundo estudo demonstraram melhora no tônus muscular dos membros superiores, do controle cervical e na qualidade do sono. Além disso, em relação ao padrão motor, verificouse aumento de $36 \%$ para $60 \%$ de itens avaliados com padrão normal/presente. Além disso, dados obtidos a partir do questionário respondido pela mãe da criança informaram melhora na qualidade do sono, no manuseio e convívio com a criança e no controle de suas pernas, uma vez que começou a esboçar alguns passos (CARVALHO R, et al., 2010). 
Um segundo estudo que deixou claro que foi realizado no ambiente domiciliar avaliou bebês de mães primíparas que receberam a massagem Shantala, no período de funcionamento da Unidade de Saúde da Família-Escola) (USF-Escola), onde foi realizada a triagem dos participantes. A metodologia de aplicação da massagem não foi bem delineada no artigo, como é possível observar no Quadro 2, mas os autores esclareceram que, para a coleta de dados, foi aplicada entrevista semiestruturada apenas no final do estudo. Os resultados apresentados no Quadro 3 incluíram: alívio de cólicas, equilíbrio no funcionamento intestinal, melhora no padrão do sono, tranquilidade e relaxamento, fortalecimento do vínculo familiar (SOUZA NR, et al., 2011).

Retornando-se para os ambientes de cuidado coletivo, um estudo realizado no Serviço de Fisioterapia Infantil (SFI) da Universidade Federal da Paraíba avaliou três crianças que receberam uma média de seis sessões de massagem Shantala aplicadas por suas mães. Das três crianças duas crianças apresentavam riscos de distúrbios no desenvolvimento motor (uma por prematuridade e a outra por disfunção motora), enquanto a terceira não apresentava história de risco no desenvolvimento motor. O Quadro 2 demonstra que a metodologia de aplicação da Shantala não foi detalhadamente descrita (MOREIRA NRTL, et al., 2011).

As informações foram coletadas a partir de entrevista semiestruturadas com as mães das crianças, no início e no término da pesquisa. Foram avaliados também relatos escritos pelas mães acerca das alterações observadas por elas em seus filhos no decorrer do estudo, de modo especial no que tange ao vínculo de mãefilho. Os resultados observados no Quadro 3 descreveram as crianças apresentavam-se tranquilas, proporcionando melhora na qualidade do sono, promovendo maior aproximação e melhora da afetividade em relação ao vínculo mãe-filho (MOREIRA NRTL, et al., 2011).

Posteriormente, outro estudo foi publicado tendo sido realizado em um Centro de Educação Infantil (CEI) da rede pública em Matinhos, Paraná. Cada bebê foi atendido por uma única acadêmica, ainda que esta pudesse atender mais de um bebê no decorrer do projeto. O Quadro 2 não mostra outros detalhes da metodologia de aplicação da Shantala além de que as crianças receberam uma média de quinze sessões de Shantala, duas vezes na semana (MOTTER AA, et al., 2012).

Antes e após o período de intervenções, as crianças foram avaliadas quanto aos antecedentes gestacionais, estado atual de saúde, desenvolvimento motor, outros itens constantes de uma ficha de avaliação. Dentre os resultados que podem ser acompanhados no Quadro 3, registrou-se o relaxamento dos bebês e o incentivo à criação e fortalecimento de vínculo com as discentes que aplicavam a massagem. Ademais, o Quadro 3 mostra que houve relatos de pais que, tendo realizado a Shantala em seus bebês em casa, observaram que eles ficavam mais tranquilos após a massagem (MOTTER AA, et al., 2012).

Finalmente, dois estudos descreveram intervenções com Shantala em ambiente de creche pública. $O$ primeiro avaliou a variação dos níveis de cortisol ao longo do dia durante uma semana em uma população de 11 bebês saudáveis. De acordo como se observa no Quadro 2, esses bebês receberam da própria autora do estudo uma sessão diária de quinze minutos cada uma, em três dias diferentes, no decorrer de uma semana. Os resultados apresentados no Quadro 3 associaram a aplicação da massagem a uma modificação nos valores de cortisol salivar, evidenciando uma possível adaptação do eixo HPA e do ritmo circadiano dos bebês envolvidos no estudo (FOGAÇA MDC, et al., 2005).

Por outro lado, o Quadro 2 apresenta que o segundo avaliou frequência cardíaca, frequência respiratória e temperatura corporal de nove latentes no primeiro ano de vida que receberam uma única sessão de Shantala em um único dia, igualmente da pesquisadora responsável pelo estudo. Foi verificada uma diferença significativa na frequência respiratória e na frequência cardíaca dos bebês que receberam a Shantala, como apresenta o Quadro 3. Esses dados puderam evidenciar a relação entre a aplicação da massagem Shantala e os sinais vitais dos bebês que participaram do estudo (LINKEVIEIUS TAK, 2012).

Ao longo dos artigos expostos acima é possível observar os efeitos benéficos da Shantala em diferentes ambientes, desde o domiciliar até aqueles de cuidado coletivo, incluindo contexto ambulatorial e escolar. (BARBOSA KC, et al., 2011; CARVALHO R, et al., 2010; FOGAÇA MDC, et al., 2005; LINKEVIEIUS TAK, et al., 2012; MOREIRA NRTL, et al., 2011; MOTTER AA, et al., 2012; SOUZA NR, et al., 2011). 
De modo geral, os estudos realizados têm mostrado que a Shantala promove o relaxamento muscular (BARBOSA KC, et al., 2011; CARVALHO R, et al., 2010) e melhora no padrão de sono das crianças que receberam essa massagem (BARBOSA KC, et al., 2011; CARVALHO R, et al., 2010; MOREIRA NRTL, et al., 2011; SOUZA NR, et al., 2011), bem como o estreitamento do vínculo de afeto com os pais e/ou profissionais que aplicaram a massagem (BARBOSA KC, et al., 2011, CARVALHO R, et al., 2010; MOREIRA NRTL, et al., 2011; MOTTER AA, et al., 2012, SOUZA NR, et al., 2011).

Esses foram os pontos que se repetiram nos estudos revisados neste trabalho. Até mesmo o melhoramento no desenvolvimento motor de crianças com histórico clínico de deficiência foi observado entre os relatos coletados (MOREIRA NRTL, et al., 2011). O alívio de cólicas e a melhora da função intestinal também foram referidos (SOUZA NR, et al., 2011), bem como modificações nos padrões de sinais vitais, como frequência cardíaca (FC) e frequência respiratória (FR) (LINKEVIEIUS TAK, et al., 2012) e marcadores bioquímicos do estresse, como o cortisol salivar (FOGAÇA MDC, et al., 2005), por exemplo.

De maneira geral, os nociceptores presentes no tecido cutâneo recebem os toques da Shantala, e ativam as sequências segmentares de neurônios aferentes, cujos estímulos alcançam o eixo hipotálamo-hipofisário via medula espinhal. Em resposta, essa cascata devolve à corrente sanguínea e aos demais tecidos neurohormônios, tais como endorfinas e encefalinas, que exercem efeitos de relaxamento e de bem-estar ao organismo como um todo, o que leva, subsequentemente, à redução dos níveis séricos de cortisol (FOGAÇA MDC, et al., 2005, GUYTON AC e HALL JE, 2006). A sensação de segurança e afeto gerados nesses bebês pelos benefícios da Shantala pode, portanto, favorecer o seu desenvolvimento físico, cognitivo e emocional (BÁRCIA S, SÁ E, 2010; BRÊTAS J, SILVA M, 1998; CARVALHO R, et al., 2010; LEBOYER F, 1995).

Um ensaio teórico realizado por Cavalcante LIC e Magalhães CMC (2012) destaca a importância do tempo de convívio entre a criança e suas figuras de referência, sejam elas da sua família de origem, ou da instituição de acolhimento, antes de encontrarem suas famílias adotivas. $O$ ensaio elenca referencial bibliográfico acerca dessa temática no intuito de trazer a discussão sobre como a formação do apego em crianças com histórico de institucionalização pode ser favorecida ou inibida de acordo com o conjunto de fatores ecológicos combinados no ambiente, de modo especial a qualidade das relações com seus cuidadores primários naquele local. Deste modo, as autoras incentivam a promoção de debates os quais possam resultar em novas propostas de intervenções que proporcionem às crianças acolhidas experiências de cuidados e relações afetuosas com os seus educadores sociais e a equipe técnica do espaço onde se encontrem acolhida, visando um processo mais saudável na formação de sua capacidade de desenvolver apego seguro em suas relações futuras (CAVALCANTE LIC, MAGALHÃES CMC, 2012).

Ademais, além das crianças que recebem a Shantala, os adultos que a aplicam também podem ser beneficiados. A seguir são apresentados três estudos onde os sujeitos avaliados foram as pessoas que aplicaram a Shantala nos bebês. É possível perceber que todos apresentaram resultados satisfatórios (FARIA KC, et al., 2018, LIVINGSTON K, et al., 2009; VICTOR JK, MOREIRA TMM, 2004).

No primeiro estudo, um grupo de cuidadoras composto por mães e uma avó que aplicaram Shantala em seus bebês internados em uma Unidade de Tratamento Intensivo Neonatal (UTIN) referiu maior satisfação na sua relação com seus bebês após a aplicação da massagem. Essas cuidadoras se disseram mais satisfeitas em relação ao tempo que passavam com seus bebês e com o envolvimento no cuidado com eles. Também se declarou muito satisfeita com o programa de massagem, o que incluiu o aprendizado de novas habilidades e a interação com o professor (LIVINGSTON K, et al., 2009).

Outro estudo teve como objetivo ensinar e estimular a prática da Shantala por familiares de bebês acompanhados durante a consulta de enfermagem e avaliar essa experiência. As cuidadoras envolvidas na intervenção foram mães, avós e tias que acompanhavam seus bebês às consultas de enfermagem em uma Unidade Básica de Saúde da Família em Fortaleza, Ceará (VICTOR JF, MOREIRA TMM, 2004).

As participantes demonstraram satisfação com o aprendizado e desejo de partilhar o conhecimento apreendido com outras pessoas, tornando-se potenciais multiplicadores da prática tradicional no seio da família e da comunidade em que vivem (VICTOR JF, MOREIRA TMM, 2004). 
É importante, ainda, citar um estudo que envolveu um grupo de 19 monitoras de nove creches mantidas pela Secretaria Municipal de Educação de Minas Gerais, localizadas na zona urbana de uma cidade do interior do Estado. Essas monitoras aplicaram a Shantala nos bebês daquela instituição em sessões diárias de 30 minutos, duas vezes por semana, durante cinco semanas. Ao final das intervenções foi registrado um maior escore no domínio psicológico das monitoras, segundo a Escala de WHOQOL-Bref, evidenciando-se melhora na percepção da sua qualidade de vida (FARIA KC, et al., 2018). Esses dados demonstram que não apenas quem recebe, mas também quem aplica a Shantala pode igualmente se beneficiar dos seus efeitos.

De maneira geral, os nociceptores presentes no tecido cutâneo recebem os toques da Shantala, e ativam as sequências segmentares de neurônios aferentes, cujos estímulos alcançam o eixo hipotálamo-hipofisário via medula espinhal. Em resposta, essa cascata devolve à corrente sanguínea e aos demais tecidos neurohormônios, tais como endorfinas e encefalinas, que exercem efeitos de relaxamento e de bem-estar ao organismo como um todo, o que leva, subsequentemente, à redução dos níveis séricos de cortisol (FOGAÇA MDC, et al., 2005; GUYTON AC, HALL JE, 2006). A sensação de segurança e afeto gerados nesses bebês pelos benefícios da Shantala pode, portanto, favorecer o seu desenvolvimento físico, cognitivo e emocional (BÁRCIA S, SÁ E, 2010; BRÊTAS J, SILVA M, 1998; CARVALHO R, et al., 2010; LEBOYER F, 1995).

Reconhece-se que o toque com intenção terapêutica é um processo de comunicação não verbal, onde não há meramente um sujeito ativo e outro passivo, mas existe uma troca de estímulos mútuos que se retroalimentam entre os dois atores. O prazer e o bem-estar que o bebê expressa por receber a Shantala incentiva o cuidador no processo, o qual não se limita à simples aplicação da massagem, mas que envolve uma interação íntima e profunda entre dois seres que compartilham um aprendizado prazeroso. Deste modo, esses efeitos, podem, igualmente, contribuir para a promoção da qualidade de vida e saúde dos cuidadores dessas crianças, o que pode ser um fator a mais que os encorajem a investir seu tempo e dedicação em práticas como a Shantala (RIBEIRO-LIMA TV, et al., 2018).

\section{CONSIDERAÇÕES FINAIS}

A partir desta revisão da literatura efetuada a partir de periódicos indexados, pressupõe- se, portanto, que intervenções envolvendo a massagem Shantala realizadas no intuito de promover o bem-estar de bebês em seus primeiros meses de vida, seja uma alternativa válida para atenuar os efeitos deletérios de eventos estressores, independentemente se esta criança se encontra sob os cuidados de sua família em seu lar, ou de profissionais de saúde em ambientes de cuidado coletivo. Por outro lado, as informações encontradas acerca dos desenhos metodológicos dos estudos sobre a aplicação da Shantala em diferentes ambientes evidenciam a necessidade de elaboração e descrição mais detalhadas de suas metodologias, visando possíveis reproduções futuras para fins de comparação entre sujeitos e populações em contextos diversos. Tais intervenções se constituem como recursos terapêuticos que podem reduzir o estresse vivenciado por esses bebês, podendo auxiliar sobremaneira nos aspectos psicológico, motor, físico e emocional de seu desenvolvimento.

\section{FINANCIAMENTO}

Este estudo foi financiado pela Coordenação de Aperfeiçoamento de Pessoal de Nível Superior, de acordo com o processo ํㅡ 23038.013648/2018-51, da portaria ํㅡㄹ 206, de 4 de setembro de 2018 .

\section{REFERÊNCIAS}

1. BARBOSA KC, et al. Efeitos da shantala na interação entre mãe e criança com síndrome de down. Journal of Human Growth and Development. 2011; 21(2): 356-361.

2. BÁRCIA S e SÁ E. A importância do toque e da massagem do bebé alguns apontamentos. In: J. Carvalho (Ed.), Psicologia da Saúde. Lisboa: Climepsi. 2010;147-160.

3. BRÊTAS J e SILVA M. Massagem em bebês: um projeto de extensão comunitária. Acta Paulista de Enfermagem, 1998, 11(ESPECIAL), 59-63.

REAS/EJCH | Vol.Sup.n.48 | e2375 | DOI: https://doi.org/10.25248/reas.e2375.2020 Página 8 de 9 
4. CARVALHO R, et al. Shantala no desenvolvimento neuropsicomotor em pdortador da Síndrome de Down Autoras Palavras-chave. Revista Cientifico Do UNIFAE,. 2010; 4(1): 62-66.

5. CAVALCANTE LIC e MAGALHÃES CMC. Relações de apego no contexto da institucionalização na infância e da adoção tardia. Psicologia Argumento. 2012; 30(68), 75-85.

6. CRUZ C e CAROMANO F. Características das técnicas de massagem para Bebês. Rev. Ter. Ocup. Univ. São Paulo. 2005; 16(1): 47-53.

7. CRUZ C e CAROMANO F. Levantamento de conteúdos para fundamentação do ensino dos efeitos psicocomportamentais da massagem para bebês. Acta Fisiátrica. 2007; 14(1): 11-16.

8. FARIA KC de, et al. Impacto da qualidade de vida de monitoras de bebês institucionalizados antes e após a aplicação da massagem Shantala. Revista Brasileira de Qualidade de Vida. 2018; 10(1): 1- 12.

9. FIELD T, et al. Tactile kinesthetic stimulation effects on preterm neonates. Pediatrics. 1986; 77(5): 654-658.

10. FOGAÇA MDC, et al. Salivary cortisol as an indicator of adrenocortical function in healthy infants, using massage therapy. São Paulo, Medical Journal. 2005; 123(5): 215-218.

11. FONSECA LF, et al. Compêndio de neurologia infantil (Medsi). Rio de Janeiro. 2002.

12. GUYTON AC e HALL JE. O sistema nervoso autônomo e a medula adrenal. In J. E. Hall (Ed.), Tratado de fisiologia médica. 11th ed., Rio de Janeiro: Elsevier. 2006; 748-760.

13. LEBOYER F. SHANTALA - Uma arte tradicional - Massagem para bebês 7th ed. São Paulo: Ground. 1995.

14. LIMA PLS de. Estudo exploratório sobre os benefícios da shantala em bebês portadores de síndrome de down. Universidade Federal do Paraná. 2004.

15. LINKEVIEIUS TAK, et al. A influência da massagem shantala nos sinais vitais em lactentes no primeiro ano de vida. Revista Neurociencias. 2012; 20(4): 505-510.

16. LIVINGSTON K, et al. Touch and massage for medically fragile infants. Evidence-Based Complementary and Alternative Medicine: ECAM. 2009; 6(4): 473-482.

17. MOREIRA NRTL, et al. A percepção da mãe após aprendizado e prática do método de massagem shantala no bebê. Revista Brasileira de Ciências Da Saúde. 2011; 15(1): 25-30.

18. MOTTER AA, et al. Relato De Experiência: Shantala - massagem para bebês- Centro De Educação Infantil Rosa Maria. Divers@!..2012; 5(2): 124-136.

19. NARDO LRO, et al. Massagem shantala. Atas - Investigação Qualitativa Em Ciências Sociais. 2014; 3: $273-278$.

20. RIBEIRO-LIMA TV e CAVALCANTE LIC. A massagem infantil como recurso para modulação do estresse em bebês em ambiente de cuidados coletivos. Revista Eletrônica Acervo Saúde. 2019; 11(1): e438.

21. RIBEIRO-LIMA TV, et al. Estratégias cooperativas no contexto de acolhimento institucional: psicomotricidade relacional e massagem para bebês. Acolhimento institucional de crianças e adolescentes: teorias e evidências científicas para boas práticas, 1st ed. Curitiba: Juruá. 2018: 436.

22. SOUZA NR, et al. Shantala massagem para bebês: experiência materna e familiar. Ciencia et Praxis. 2011; 4(7): $55-60$.

23. UMEMURA JF, et al. Shantala: intervenção fisioterapêutica utilizada em bebês prematuros de baixo peso. In: $V$ Mostra Interna de Trabalhos de Iniciação Científica. Maringá, PR. 2010; 85-88.

24. VICTOR JF e MOREIRA TMM. Integrando a família no cuidado de seus bebês: ensinando a aplicação da massagem Shantala. Acta Scientiarum - Health Sciences. 2004; 26(1):35-39. 